\title{
A GEOMETRIC PROOF OF A RESULT OF TAKEUCHI
}

\author{
Peter Quast
}

(Received March 21, 2013, revised August 29, 2013)

\begin{abstract}
In 1984 Masaru Takeuchi showed that every real form of a hermitian symmetric space of compact type is a symmetric $R$-space and vice-versa. In this note we present a geometric proof of this result.
\end{abstract}

1. Introduction. Symmetric $R$-spaces can be described in several ways. An early definition of symmetric $R$-spaces by Takeuchi [19] has a slightly algebraic flavour: Symmetric $R$-spaces are compact Riemannian symmetric spaces that are also $R$-spaces (generalized flag manifolds), that is they can also be written as quotients of non-compact connected center-free semi-simple Lie groups by parabolic subgroups. Symmetric $R$-spaces are closely related to certain gradings of semi-simple Lie algebras of the form $\mathfrak{g}=\mathfrak{g}_{-1} \oplus \mathfrak{g}_{0} \oplus \mathfrak{g}_{1}$, sometimes called symmetric graded Lie algebras (see [13, 19] and [20]). The (local) classification of indecomposable symmetric $R$-spaces is due to Kobayashi and Nagano [12,13] (see also [1, p. 310f]).

There is also a more geometric description of symmetric $R$-spaces; they are $s$-orbits of extrinsically symmetric elements (see $[18,11,14,9,10]$ and Section 2 ). In this realization symmetric $R$-spaces are extrinsically symmetric submanifolds (see [4] and [6, p. 82]). Ferus has shown that this property characterizes symmetric $R$-spaces (see [5, 6, 2]): Symmetric $R$ spaces are precisely the compact extrinsically symmetric submanifolds of Euclidean spaces.

The indecomposable symmetric $R$-spaces divide into two different types:

(i) irreducible hermitian symmetric spaces of compact type;

(ii) indecomposable symmetric $R$-spaces of non-hermitian type.

In this note we give a geometric proof of Takeuchi's result:

THEOREM 1.1 (Takeuchi [20]). Every symmetric $R$-space can be realized as a real form of a hermitian symmetric space of compact type. Vice-versa every real form of a hermitian symmetric space of compact type is a symmetric $R$-space.

While Takeuchi's proof in [20] uses the algebraic description of symmetric $R$-spaces in terms of symmetric graded Lie algebras, our proof is rather based on the geometric realization of symmetric $R$-spaces as $s$-orbits of extrinsically symmetric elements, or equivalently, as compact extrinsically symmetric spaces of Euclidean spaces. The main tool in our proof is a geometric property of standardly embedded hermitian symmetric spaces of compact type

2010 Mathematics Subject Classification. Primary 32M15; Secondary 53C35, 53C40.

Key words and phrases. Hermitian symmetric spaces, symmetric $R$-spaces, real forms. 
proved in [3]. Every isometry of a standardly embedded hermitian symmetric space of compact type is the restriction of a linear isometry of the ambient space. We shall proof en passant (see Remark 3.1 and Proposition 3.2) a precised version of Takeuchi's theorem, namely:

THEOREM 1.2 (Specified version of Takeuchi's theorem). Every indecomposable nonhermitian symmetric $R$-space is a real form of an irreducible hermitian symmetric space of compact type and vice versa.

Theorem 1.2 can also be verified by comparing case-by-case Leung's classification of real forms of irreducible hermitian symmetric spaces in [15, Theorem 3.4] with the classification of indecomposable non-hermitian symmetric $R$-space (see e.g. [1, p. 311]).

We learned from the referee that yet another proof of the implication in Takeuchi's theorem we discuss in Paragraph 3.2 can be found in the recent article [21, proof of Theorem 4.3]. The proof given there uses a perspective on symmetric $R$-spaces rather similar to ours, but it is still slightly different.

\section{Preliminaries.}

2.1. Symmetric $R$-space as $s$-orbits. The classical facts about symmetric spaces used below can be found in the standard literature like Helgason's famous monograph [7] or Wolf's book [22, Part IV].

Every symmetric $R$-space arises in the following way (see [18, 11, 14, 9, 10, 4] and also [1, pp. 70-72]): Let $S$ be a symmetric space of compact type (we always assume symmetric spaces to be connected) and let $L$ be the identity component of the isometry group of $S$. The geodesic symmetry $s_{o}$ of $S$ at a chosen base point $o \in S$ gives rise to an involutive Lie group automorphism

$$
\sigma: L \rightarrow L, \quad l \mapsto s_{o} \circ l \circ s_{o} .
$$

The differential $\sigma_{*}$ of $\sigma$ at the identity is therefore an involutive automorphism of the Lie algebra $\mathfrak{l}$ of $L$, called the Cartan involution of $(S, o)$. We denote by $\mathfrak{h}$ the fixed point set of $\sigma_{*}$ and by $\mathfrak{s}$ its $(-1)$-eigenspace. The decomposition

$$
\mathfrak{l}=\mathfrak{h} \oplus \mathfrak{s}
$$

called Cartan decomposition of $\mathfrak{l}$ corresponding to $(S, o)$, is orthogonal w.r.t. the CartanKilling form $B_{\mathfrak{l}}$ of $\mathfrak{l}$. This decomposition satisfies the Cartan relations, namely

$$
[\mathfrak{h}, \mathfrak{h}] \subset \mathfrak{h}, \quad[\mathfrak{h}, \mathfrak{s}] \subset \mathfrak{s} \quad \text { and } \quad[\mathfrak{s}, \mathfrak{s}] \subset \mathfrak{h} .
$$

The Lie subalgebra $\mathfrak{h} \subset \mathfrak{g}$ is the Lie algebra of the identity component $H$ of the isotropy group of $o$ in $G$. Moreover, $\mathfrak{s}$ can be identified with the tangent space $T_{o} S$ by the restriction of the differential at the identity of the projection of the principal bundle $L \rightarrow S, l \mapsto l . o$. Here l.o denotes the action of the isometry $l$ of $S$ on the point $o \in S$. Using the above identification $\mathfrak{s} \cong T_{o} S$, the linear isotropy action of $H$ on $T_{o} S$, also known as $s$-representation, becomes the restriction of the adjoint action:

$$
H \times \mathfrak{s} \rightarrow \mathfrak{s}, \quad(h, X) \mapsto \operatorname{Ad}_{L}(h) X .
$$


A non-zero element $\xi \in \mathfrak{s}$ is called extrinsically symmetric (or minuscule coweight), if

$$
\operatorname{ad}_{\mathfrak{l}}(\xi)^{3}=-\operatorname{ad}_{\mathfrak{l}}(\xi),
$$

or equivalently, if the eigenvalue spectrum of $\operatorname{ad}(\xi)$ equals $\{-i, 0, i\}$. We may assume w.r.g. that no projection of $\xi$ onto a simple factor of $\mathfrak{l}$ vanishes.

A symmetric $R$-space is an isotropy orbit (s-orbit)

$$
M:=\operatorname{Ad}_{L}(H) \xi \subset \mathfrak{s},
$$

of $S$ where $\xi \in \mathfrak{s}$ is an extrinsically symmetric element. Ferus has shown that $M$ is an extrinsically symmetric submanifold of the Euclidean space $\mathfrak{s}$ (see [4] and [6, p. 82]). We call $M$ indecomposable if $S$ is an irreducible symmetric space of compact type. If $S$ is an irreducible symmetric space of compact type, but not a compact simple Lie group, $M$ is an indecomposable symmetric $R$-space of non-hermitian type (see e.g. [1, p. 310f.]). For a description of extrinsically symmetric elements in terms of roots we refer to [17, Lemma 2.1] and also to [12, Section 6].

2.2. Hermitian symmetric spaces of compact type as $R$-spaces. If $S=G$ is a compact connected semi-simple center-free Lie group, then $L$ is isomorphic to $G \times G$, and the linear isotropy representation on the tangent space $T_{e} G$ is equivalent to the adjoint representation of $G$ on $\mathfrak{g}$ (see e.g. [7, $\$ 6$ of Chapter IV]).

Let $\xi \in \mathfrak{g}$ be extrinsically symmetric. It is well-known that $P:=\operatorname{Ad}(G) \xi \subset \mathfrak{g}$ endowed with the Riemannian metric induced by the scalar product $-B_{\mathfrak{g}}$ on $\mathfrak{g}$ is a hermitian symmetric space of compact type (see [8]). Let $X \in P$, then $\operatorname{Ad}(\exp (\pi / 2 \cdot X))$ and $\operatorname{ad}(X)$ coincide on $T_{X} P \subset \mathfrak{g}$ and they define a Kähler structure $J_{X}$ of $P$ at the point $X$, that is

$$
J_{X}=\left.\operatorname{Ad}(\exp (\pi / 2 \cdot X))\right|_{T_{X} P}=\left.\operatorname{ad}(X)\right|_{T_{X} P},
$$

which turns $P$ into a hermitian symmetric space.

The geodesic symmetry $s_{X}$ of $P$ at the point $X$ extends to the reflection $\rho_{X}$ of $\mathfrak{g}$ along the normal space $N_{X} P=\{Y \in \mathfrak{g} ; \operatorname{ad}(X) Y=0\}$ given by the involutive automorphism

$$
\rho_{X}:=\operatorname{Ad}(\exp (\pi X))
$$

of $\mathfrak{g}$. Finally, if we assume that all projections of $\xi$ onto simple factors of $\mathfrak{g}$ are non-zero, $G$ can be identified with the identity component of the isometry group of $P$.

Conversely every hermitian symmetric space $P$ of compact type can be realized as such an orbit in the Lie algebra of its infinitesimal isometries (see [16, pp. $165 \mathrm{ff}$.] and [8]). If we endow this Lie algebra with a scalar product that coincides on each irreducible factor with the Cartan-Killing form up to a suitable negative constant, this embedding is isometric. We call this the standard embedding of a hermitian symmetric space of compact type.

2.3. Real forms of hermitian symmetric spaces. Following Takeuchi [20], a real form of a hermitian symmetric space $P$ is a connected component of the fixed point set of some involutive and anti-holomorphic isometry $f$ of $P$. Real forms are totally geodesic halfdimensional real submanifolds of $P$. 
3. The proof. In this section we present a geometric proof of Takeuchi's result, Theorem 1.1 (see [20]). We show both implications in Takeuchi's theorem separately.

3.1. The proof of the first implication. The arguments given in this paragraph are classical and straightforward. They may also be adapted to more general situations.

Let $S$ be a symmetric space of compact type, $o \in S$ a base point, $\sigma_{*}$ the corresponding Cartan involution and $\mathfrak{l}=\mathfrak{h} \oplus \mathfrak{s}$ the induced Cartan decomposition of the semi-simple Lie algebra $\mathfrak{l}$ of infinitesimal isometries of $S$. Let $\xi \in \mathfrak{s}$ be an extrinsically symmetric element and $M:=\operatorname{Ad}_{L}(H) \xi$ a symmetric $R$-space. We may again assume that no projection of $\xi$ onto a simple factor of $l$ is zero. The inclusion $H \hookrightarrow L$ of the identity component $H$ of the isotropy group of $o$ into the identity component $L$ of the full isometry group of $S$ provides a natural inclusion

$$
\mathfrak{s} \supset M=\operatorname{Ad}_{L}(H) \xi \hookrightarrow \operatorname{Ad}_{L}(L) \xi=: P \subset \mathfrak{l}
$$

of the symmetric $R$-space $M$ into the hermitian symmetric space $P$.

The linear automorphism $F:=-\sigma_{*}$ of $\mathfrak{l}$ preserves the scalar product on $\mathfrak{l}$ and maps adjoint orbits onto adjoint orbits. Since $\xi$ lies in $\mathfrak{s}$, the $(-1)$-eigenspace of $\sigma_{*}, \xi$ is a fixed point of $F$. Thus $F$ leaves $P$ invariant and $f:=\left.F\right|_{P}$ is an involutive isometry of $P$. Let $f_{*}$ denote the differential of $f$ at the fixed point $\xi$. To show that $f$ is anti-holomorphic, it is sufficient to verify that $f_{*}\left(J_{\xi} X\right)=-J_{\xi} f_{*}(X)$ for all $X \in T_{\xi} P$, because the complex structure $J$ of $P$ is parallel. Equation (1) implies

$$
\begin{aligned}
f_{*}\left(J_{\xi} X\right) & =F[\xi, X]=-\sigma_{*}[\xi, X]=-\left[\sigma_{*} \xi, \sigma_{*} X\right] \\
& =\left[\xi, \sigma_{*} X\right]=-[\xi, F X]=-J_{\xi} f_{*}(X) .
\end{aligned}
$$

Since $T_{\xi} P \subset \mathfrak{l}$ is the $(-1)$-eigenspace of $(\operatorname{ad}(\xi))^{2}$ and since $(\operatorname{ad}(\xi))^{2}$ commutes with $F$, we see that of $T_{\xi} M=\left\{X \in \mathfrak{s} ;(\operatorname{ad}(\xi))^{2}(X)=-X\right\}=T_{\xi} P \cap \mathfrak{s}$ (see also e.g. [1, p. 71]). Thus $M$ is a connected component of the fixed point set of $f$. This shows that $M$ is a real form of $P$.

REMARK 3.1. If $M$ is an indecomposable symmetric $R$-space, that is, if $S$ is an irreducible symmetric space of compact type, but not a compact Lie group, or equivalently, if $\mathfrak{l}$ is a simple compact Lie algebra, then $P$ is an irreducible hermitian symmetric space of compact type.

3.2. The proof of the converse implication. We now show the converse impliciation in Takeuchi's theorem, namely that every real form of a hermitian symmetric space $P$ of compact type is a symmetric $R$-space. As a major tool we use the results of Eschenburg, Tanaka and the author on the extension of isometries of standardly embedded hermitian symmetric spaces published in [3]. The referee kindly informed us that a proof of this implication in Takeuchi's theorem using slightly different arguments can be found in [21, proof of Theorem 4.3].

Since a hermitian symmetric space $P$ of compact type is simply connected (see e.g. [7, Theorem 4.6 in Chapter VIII]), $P$ is a product of its irreducible de Rham factors

$$
P=P_{1} \times \cdots \times P_{k},
$$


where each factor is an irreducible hermitian symmetric space of compact type (see also [22, Corollary 8.7.11]). An involutive anti-holomorphic isometry $f$ either preserves a de Rham factor or permutes isometric de Rham factors pairwise. Thus it is sufficient to only consider the following two cases:

(I) $P$ is the Riemannian product of two equal irreducible hermitian symmetric spaces $Q$ of compact type, that is $P=Q \times Q$, and $f$ permutes both factors.

(II) $P$ is irreducible.

We start by investigating the first case. Let $\tau$ denote the isometry of $P=Q \times Q$ that just interchanges both factors, that is $\tau(x, y)=(y, x)$ for all $x, y \in Q$. Then $f$ has the form $f=\left(f_{1} \times f_{2}\right) \circ \tau$, where $f_{1}$ and $f_{2}$ are anti-holomorphic isometries of $Q$. Since $f$ is involutive, we get $f_{2}=f_{1}^{-1}$, that is $f=\left(f_{1} \times f_{1}^{-1}\right) \circ \tau$. The fixed point set of $f$,

$$
\{(x, y) \in P ; f(x, y)=(x, y)\}=\left\{\left(x, f_{1}^{-1}(x)\right) ; x \in Q\right\},
$$

is isomorphic to $Q$ and hence a symmetric $R$-space.

To treat the second case we prove the following statement:

PROPOSITION 3.2. Every real form of an irreducible hermitian symmetric space $P$ of compact type is an indecomposable symmetric $R$-space of non-hermitian type.

If $P=\operatorname{Ad}(G) \xi \subset \mathfrak{g}$ is a standardly embedded irreducible hermitian symmetric space of compact type, then the Lie algebra $\mathfrak{g}$ of its infinitesimal isometries is simple (see e.g. [7, $\S 6$ in Chapter VIII]). We consider $\mathfrak{g}$ endowed with the scalar product that coincides with the Cartan-Killing form $B_{\mathfrak{g}}$ up to a negative factor. The Cartan involution corresponding to $(P, \xi)$ is $\rho_{\xi}$ given in Equation (2). The induced Cartan decomposition is $\mathfrak{g}=\mathfrak{k} \oplus \mathfrak{p}$, where $\mathfrak{k}$ is the fixed point set of $\rho_{\xi}$.

Let $f$ be an involutive anti-holomorphic isometry of $P$ and let $M$ be a non-empty connected component of the fixed point set of $f$. Then $M$ is a real form of $P$ and we must show that $M$ is an indecomposable symmetric $R$-space of non-hermitian type. By the homogeneity of $P$ we may assume w.r.g. that $\xi$ is a point of $M$ and therefore $f(\xi)=\xi$.

The differential $f_{*}$ of $f$ at $\xi$ is an involutive linear automorphism of $\mathfrak{p} \cong T_{\xi} P$. The fixed point set $\mathfrak{m}$ of $f_{*}$ is canonically identified with the tangent space $T_{\xi} M$.

Following the reasoning in [3, Section 3] we consider the Lie group automorphism

$$
\phi: G \rightarrow G, \quad g \mapsto f \circ g \circ f
$$

of the identity component $G$ of the full isometry group of $P$. Since $\phi$ leaves the stabilizer $K$ of $\xi$ in $G$ invariant, its differential $\phi_{*}$ at the identity induces an automorphism of $\mathfrak{k}$. We conclude (see [3, Lemma 3.1]) that

$$
\phi_{*}(\xi) \in\{ \pm \xi\} .
$$

LEMMA 3.3. We have $\phi_{*}(\xi)=-\xi$.

Proof. Assume by contradiction that $\phi_{*}(\xi)=\xi$. Then the derivative of the oneparameter family

$$
\boldsymbol{R} \rightarrow G, s \mapsto \phi(\exp (s \cdot \xi))=f \circ \exp (s \cdot \xi) \circ f
$$


at $s=0$ is $\phi_{*}(\xi)=\xi$. Hence

$$
\exp (s \cdot \xi)=f \circ \exp (s \cdot \xi) \circ f \text { for all } s \in \boldsymbol{R} .
$$

Let $\gamma$ be the geodesic in $M \subset \mathfrak{g}$ that satisfies $\gamma(0)=\xi$ and $\dot{\gamma}(0)=: X \in \mathfrak{m} \backslash\{0\}$. Taking $s=\frac{\pi}{2}$ we get

$$
\exp \left(\frac{\pi}{2} \xi\right) \cdot \gamma(t)=\left(f \circ \exp \left(\frac{\pi}{2} \xi\right) \circ f\right) \cdot \gamma(t)=\left(f \circ \exp \left(\frac{\pi}{2} \xi\right)\right) \cdot \gamma(t) .
$$

The derivative at $t=0$ yields

$$
\begin{aligned}
& \left(f_{*} \circ d\left(\exp \left(\frac{\pi}{2} \cdot \xi\right)\right)_{\xi}\right) X=f_{*}\left(\operatorname{Ad}\left(\exp \left(\frac{\pi}{2} \cdot \xi\right)\right) X\right) \\
= & f_{*}\left(J_{\xi} X\right)=d\left(\exp \left(\frac{\pi}{2} \cdot \xi\right)\right)_{\xi} X=\operatorname{Ad}\left(\exp \left(\frac{\pi}{2} \cdot \xi\right)\right) X=J_{\xi} X
\end{aligned}
$$

(see Equation (1)). But the equation $f_{*}\left(J_{\xi} X\right)=J_{\xi} X=J_{\xi} f_{*}(X)$ for a nonzero $X \in \mathfrak{m} \cong$ $T_{\xi} M$ contradicts the fact that $f$ is anti-holomorphic.

Notice that the fact $\phi_{*}(\xi)=-\xi$ also plays a role in [21, proof of Theorem 4.3].

The proof of the main result in [3] shows that in our case $f$ is the restriction to $P$ of the linear isometry

$$
F:=-\phi_{*}: \mathfrak{g} \rightarrow \mathfrak{g} .
$$

LEMMA 3.4. $\phi_{*}=-F$ is an involutive automorphism of $\mathfrak{g}$ that commutes with $\rho_{\xi}$.

Proof. Recall that $\phi_{*}$ preserves $\mathfrak{k}$ and therefore also $\mathfrak{p}$. Notice further that $\rho_{\xi}=$ $\operatorname{Ad}(\exp (\pi \xi))$ is the identity on $\mathfrak{k}$ and $-\operatorname{Id}$ on $\mathfrak{p}$. This shows the claim.

Thus $\left(\mathfrak{g}, \phi_{*}\right)$ is an orthogonal involutive Lie algebra (see e.g. [22, Chapter 8]). Let $\mathfrak{h}$ be the fixed point set of $\phi_{*}$ and $\mathfrak{s}$ the fixed point set of $F=-\phi_{*}$. Then the orthogonal decomposition

$$
\mathfrak{g}=\mathfrak{h} \oplus \mathfrak{s}
$$

is the Cartan decomposition of some irreducible pointed symmetric space $S$ of compact type (see e.g. [22, Section 8.3]), which is not a compact Lie group (see e.g. [7, p. 379]).

Moreover, since $\phi_{*}$ and $\rho_{\xi}$ commute, we get a common eigenspace decomposition

$$
\mathfrak{g}=\mathfrak{k}_{+} \oplus \mathfrak{k}_{-} \oplus \mathfrak{p}_{-} \oplus \mathfrak{p}_{+},
$$

where $\mathfrak{k}=\mathfrak{k}_{+} \oplus \mathfrak{k}_{-}, \mathfrak{p}=\mathfrak{p}_{-} \oplus \mathfrak{p}_{+}, \mathfrak{h}=\mathfrak{k}_{+} \oplus \mathfrak{p}_{+}$and $\mathfrak{s}=\mathfrak{k}_{-} \oplus \mathfrak{p}_{-}$. Notice that $\xi \in \mathfrak{k}_{-} \subset \mathfrak{s}$ and that $\mathfrak{m}=\mathfrak{p} \cap \mathfrak{s}=\mathfrak{p}_{-}$.

We observe that $M$ is the connected component of $P \cap \mathfrak{s}$ that contains $\xi$. Let $H$ be the identity component of the closed subgroup of $G$ formed by all elements $g \in G$ enjoying the property $\operatorname{Ad}_{G}(g) \mathfrak{s}=\mathfrak{s}$. Since the decomposition $\mathfrak{g}=\mathfrak{h} \oplus \mathfrak{s}$ is orthogonal, we get $\operatorname{Ad}_{G}(h) \mathfrak{h}=$ $\mathfrak{h}$ for all $h \in H$. One easily checks that $\mathfrak{h}$ is the Lie algebra of $H$.

Since the representation $\left.\operatorname{Ad}_{G}(H)\right|_{\mathfrak{s}}$ is the $s$-representation of the irreducible symmetric space $G / H$ of compact type, which is not a compact Lie group, the following Lemma implies Proposition 3.2: 
LEMMA 3.5. The real form $M$ is the orbit $M=\operatorname{Ad}_{G}(H) \xi$.

Proof. The inclusion $\operatorname{Ad}_{G}(H) \xi \subset M$ is evident. Since both $M$ and $\operatorname{Ad}_{G}(H) \xi$ are connected compact submanifolds of $P$ without boundary, it now suffices to show that the dimensions of $M$ and $\operatorname{Ad}_{G}(H) \xi$ coincide.

The Lie algebra of the stabilizer of $\xi$ in $H$ is $\mathfrak{k}_{+}=\{X \in \mathfrak{h} ; \operatorname{ad}(X) \xi=0\}$ and therefore $\operatorname{dim}\left(\operatorname{Ad}_{G}(H) \xi\right)=\operatorname{dim}\left(\mathfrak{p}_{+}\right)$. On the other hand we have $\operatorname{dim}(M)=\operatorname{dim}(\mathfrak{m})=\operatorname{dim}\left(\mathfrak{p}_{-}\right)$. The automorphism $\operatorname{Ad}(\exp (\pi / 2 \cdot \xi))$ of $\mathfrak{g}$, which coincides on $\mathfrak{p}$ with $J_{\xi}$ (see Equation (1)), exchanges $\mathfrak{p}_{-}$and $\mathfrak{p}_{+}$. Indeed for $X \in \mathfrak{p}_{ \pm}$we get:

$$
\begin{aligned}
\phi_{*}\left(\operatorname{Ad}\left(\exp \left(\frac{\pi}{2} \cdot \xi\right)\right) X\right) & =\operatorname{Ad}\left(\exp \left(\frac{\pi}{2} \cdot \phi_{*}(\xi)\right)\right) \phi_{*}(X) \\
& = \pm \operatorname{Ad}\left(\exp \left(-\frac{\pi}{2} \cdot \xi\right)\right) X \\
& = \pm \operatorname{Ad}\left(\exp \left(\frac{\pi}{2} \cdot \xi\right)\right)(\operatorname{Ad}(\exp (-\pi \cdot \xi)) X) \\
& = \pm \operatorname{Ad}\left(\exp \left(\frac{\pi}{2} \cdot \xi\right)\right)(\operatorname{Ad}(\exp (\pi \cdot \xi)) X) \\
& =\mp \operatorname{Ad}\left(\exp \left(\frac{\pi}{2} \cdot \xi\right)\right) X .
\end{aligned}
$$

In the last equality we used Equation (2).

\section{REFERENCES}

[1] J. Berndt, S. Console And C. Olmos, Submanifolds and holonomy, Chapman Hall/CRC Res. Notes in Math. 434, Chapman \& Hall/CRC, Boca Raton, FL, 2003.

[2] J.-H. Eschenburg And E. Heintze, Extrinsic symmetric spaces and orbits of $s$-representations, Manuscripta Math. 88 (1995), 517-524.

[ 3 ] J.-H. Eschenburg, P. QUAST AND M. S. TANAKA, Isometries of hermitian symmetric spaces, J. Lie Theory 23 (2013), 113-118.

[4] D. FERUs, Immersionen mit paralleler zweiter Fundamentalform: Beispiele und Nicht-Beispiele., Manuscripta Math. 12 (1974), 153-162.

[ 5 ] D. FerUS, Immersions with parallel second fundamental form, Math. Z. 140 (1974), 87-93.

[6] D. FERUs, Symmetric submanifolds of euclidean space, Math. Ann. 247 (1980), 81-93.

[ 7 ] S. Helgason, Differenital geometry, Lie groups and symmetric spaces, Pure Appl. Math. 80, Academic Press, New York, 1978.

[ 8 ] U. HiRZEBRUCH, Über eine Realisierung der hermiteschen, symmetrischen Räume, Math. Z. 115 (1970), 371-382.

[9] E. KelLy, Tight equivariant immersions of symmetric spaces, Bull. Amer. Math. Soc. 77 (1971), 580-583.

[10] E. KeLly, Tight equivariant imbeddings of symmetric spaces, J. Differential Geom. 7 (1972), 535-548.

[11] S. KobaYASHI, Isometric imbeddings of compact symmetric spaces, Tohoku Math. J. (2) 20 (1968), 21-25.

[12] S. Kobayashi and T. Nagano, On filtered Lie algebras and geometric structures I, J. Math. Mech. 13 (1964), 875-907.

[13] S. Kobayashi And T. Nagano, On filtered Lie algebras und geometric structures II, J. Math. Mech. 14 (1965), 513-521.

[14] S. Kobayashi And M. Takeuchi, Minimal imbeddings of $R$-spaces, J. Differential Geom. 2 (1968), 203215. 
[15] D. S. P. LEUNG, Reflective submanifolds. IV. Classification of real forms of hermitian symmetric spaces, J. Differential Geom. 14 (1979), 179-185.

[16] A. Lichnérowicz, Géométrie des groupes de transformations, Travaux et Recherches Mathématiques, III. Dunod, Paris, 1958.

[17] A.-L. MARE, AND P. QUAST, On some spaces of minimal geodesics in Riemannian symmetric spaces, Q. J. Math. 63 (2012), 681-694.

[18] T. NAgano, Transformation groups on compact symmetric spaces, Trans. Amer. Math. Soc. 118 (1965), 428-453.

[19] M. TAKeUchi, Cell decompositions and Morse equalities on certain symmetric spaces, J. Fac. Sci., Univ. Tokyo, Sect. I 12 (1965), 81-191.

[20] M. TAKEUCHI, Stability of certain minimal submanifolds of compact Hermitian symmetric spaces, Tohoku Math. J. (2) Ser. 36 (1984), 293-314.

[21] M. S. TANAKA AND H. TASAKi, Antipodal sets of symmetric $R$-spaces, Osaka J. Math. 50 (2013), 161-169.

[22] J. A. Wolf, Spaces of constant curvature, 5th edition, Publish or Perish Inc., Houston, TX, 1984.

INSTITUT FÜR MATHEMATIK

UNIVERSITÄT AUgSBURG

86135 AUGSBURG

GERMANY

E-mail address: peter.quast@math.uni-augsburg.de 